\title{
WEIGHTED CONDITIONAL EXPECTATION OPERATORS
}

\author{
JOHN DAVID HERRON
}

\begin{abstract}
This paper presents the fundamental operator-theoretic properties of products of conditional expectation and multiplication operators. It is shown that boundedness of such a product need not depend on the boundedness of the multiplication operator. The spectrum is described, as is the unique polar decomposition. It is also shown that compactness implies the existence of an atom in the underlying $\sigma$-subalgebra. An algebra containing such operators is shown to be weakly closed and, when the underlying space is of finite measure, its commutant is an algebra of multiplication operators with suitably measurable symbol.
\end{abstract}

Mathematics subject classification (2010): Primary 47A15.

Keywords and phrases: Conditional expectation, multiplication operator.

\section{REFERENCES}

[1] C. Burnap, I.B. Jung, AND A. LAmbert, Separating partial normality classes with composition operators, J. Operator Theory, 53, 2 (2005), 381-397.

[2] R. Douglas, Contractive projections on an $L^{1}$ space, Pacific J. Math., 15, 2 (1965), 443-462.

[3] J. Grobler; B. De PAGTER, Operators representable as multiplication-conditional expectation operators, J. Operator Theory, 48 (2002), 15-40.

[4] J. GrobleR; D. Rambane, Operators represented by conditional expectations and random measures, Positivity, 9 (2005), 369-383.

[5] A. LAMBERT, A Hilbert $C^{*}$-module view of some spaces related to probabilistic conditional expectation, Quaestiones Mathematicae, 22 (1999), 165-170. 